\title{
Crisis-management, Anti-stigma, and Mental Health Literacy Program for University Students (CAMPUS): Evaluating longitudinal efficacy for reducing suicide risk
}

\author{
Asumi Takahashi ${ }^{\mathrm{a}}$, Hirokazu Tachikawa ${ }^{\mathrm{b} *}$, Ayumi Takayashiki ${ }^{\mathrm{c}}$, Takami \\ Maeno $^{\mathrm{c}}$, Yuki Shiratori ${ }^{\mathrm{d}}$, Asaki Matsuzaki ${ }^{\mathrm{d}}$, Tetsuaki Arai ${ }^{\mathrm{d}}$ \\ ${ }^{a}$ School of Humanities, Hokusei Gakuen University, Hokkaido, Japan, ${ }^{b}$ Department of \\ Disaster and Community Psychiatry, Faculty of Medicine, University of Tsukuba, \\ Ibaraki, Japan, ${ }^{c}$ Department of Primary Care and Medical Education, Faculty of \\ Medicine, University of Tsukuba, Ibaraki, Japan, ${ }^{d}$ Department of Psychiatry, Faculty of \\ Medicine, University of Tsukuba, Ibaraki, Japan
}

* Correspondence: Hirokazu Tachikawa, MD, PhD, Department of Disaster and Community Psychiatry, University of Tsukuba, Tennnodai 1-1-1, Tsukuba city, Ibaraki 305-8575, Japan. E-mail: tachikawa@md.tsukuba.ac.jp. TEL/FAX: 029-873-3343 
Background: University students have specific risk factors for suicide, necessitating targeted prevention programs. This longitudinal study evaluated the efficacy of the Crisis-management, Anti-stigma, Mental health literacy Program for University Students (CAMPUS) for reduction of risk factors and promotion of preventative behaviors.

Methods: A total of 136 medical students attended the CAMPUS as a required course at the University of Tsukuba. The CAMPUS consisted of a lecture and two group sessions covering mental health literacy, self-stigma, and gatekeeper efficacy (e.g., identifying and helping at-risk individuals). The students were asked to role-play based on a movie about gatekeepers and scripts about selfstigma and suicide-related issues. Participants completed questionnaires on suicidal behavior, depression, help-seeking intentions, self-efficacy as gatekeepers, self-concealment, and self-acceptance.

Results: A total of 121 students completed the questionnaires pre- and postprogram, and 107 students also responded 6 months later. Higher-risk students demonstrated significantly reduced suicide risk 6 months post-program compared to before the program. In addition, gatekeeper self-efficacy, help-seeking intentions for formal resources, and self-acceptance were improved in both lowand higher-risk students 6 month after the program.

Limitations: The CAMPUS program was delivered as a mandatory requirement to a group with high scholastic abilities and relatively low suicide risk. Further studies are required to assess its suitability for the general university student population.

Conclusions: The CAMPUS proved effective at reducing risk factors for suicide and promoting preventative behaviors among university students.

Keywords: suicide prevention; suicide prevention education; university students 


\section{Introduction}

Suicide is the leading cause of death among Japanese university students, with about 350 dying by suicide each year. Between $10 \%$ and $40 \%$ of university students report thoughts of suicide, substantially higher than among the general population (Jain et al., 2012; Osama et al., 2014; Peltzer et al., 2017; Sun et al., 2017). Risk factors for suicide among university students include poor academic performance, pre-existing mental disorders exacerbated by stress, a history of adverse childhood experiences, smoking, alcohol and drug use, and rejection by family or colleagues (Jain et al., 2012; Osama, 2014; Zheng and Wang, 2014; Peltzer, 2017). Many of these factors are specific to the university environment, so programs tailored to this population are required for suicide prevention.

Many universities already have or are instituting suicide prevention programs. For example, an online psychoeducational program called ProHelp was shown to improve participants' help-seeking attitudes and suicide literacy (Han et al., 2018). An online program including multiple technological components, including a website and two social media networking applications, was also reported as useful by a wide spectrum of students (Manning and VanDeusen, 2011). Gatekeeper training has been shown to help students identify colleagues at risk of suicide and provide help such as referral to mental health services (Kibler and Haberyan, 2008; Indelicato et al., 2011). In Japan, Katsumata et al. (2017) reported that a 4-h suicide prevention education program improved attitudes toward suicide and an attitude needed in a peer support towards selfdestructive behaviors.

However, these studies did not evaluate direct effects on suicidal ideation and behavior. Harrod et al. (2014) reviewed eight studies on primary prevention of suicide among university students and concluded that policy interventions including means 
restriction lowered the suicide incidence compared to universities without such interventions (Joffe, 2008). However, they also concluded that studies examining the effects of classroom instruction on suicidal behavior or long-term outcomes are still required. A suicide prevention education program for high school students, the Youth Aware Mental Health (YAM), was reported to reduce suicidal ideation and suicide attempts at 12 months post-program (although not after 3 months) compared to a control group (Wasserman et al., 2015). As programs may show only transient benefits or not help those at higher risk, it is important to examine the intermediate and longer-term efficacy for prevention of suicidal behaviors and promotion of preventative behaviors.

The university-targeted mental health education program Crisis-management, Antistigma, Mental Health Literacy Program for University Students (CAMPUS) consists of a lecture, role-play sessions, and discussion modules focusing on three main components of suicide prevention: 1) mental health literacy, 2) anti-stigma, and (3) crisis-management. Mental health literacy is defined by Jorm et al. (1997) as "knowledge and beliefs about mental disorders which aid their recognition, management or prevention." Mental health literacy can improve mental health among college students (Rafal et al., 2018) and is positively correlated with help-seeking behavior (Gorczynski et al., 2017). The CAMPUS focuses on mental health literacy particularly pertinent to university students and adolescents, including stress, depression, suicidal thoughts, self-care, and support resources. The anti-stigma component aims to combat self-stigma and provide coping methods (Corrigan and Watson, 2002; Goffman, 1990). Several studies have found that people with strong self-stigma regarding mental health issues tend to have low self-esteem, low self-efficacy, and low help-seeking intention (Corrigan and Watson, 2002; Pattyn et al., 2014). The CAMPUS also addresses misunderstandings about suicide and the reasons for acquiring self-stigma 
such as experiences of discrimination, and aims to mitigate self-stigma through psychological education and exercises. Crisis management concerns gatekeeping for suicide prevention. Students learn about risk assessment, listening to those at risk, and referral to support resources. This content is referred to as gatekeeper training and is a common component of suicide prevention programs in Japan (Hashimoto et al., 2016). Although we completed CAMPUS development in 2019, we have not yet evaluated it mid-term and longer-term efficacy.

This study aimed to assess the longitudinal efficacy of CAMPUS for reduction of suicide risk factors and promotion of preventative behaviors and gatekeeper functions among medical students. We speculated that this program would reduce suicide risk among participants at higher risk and enhance preventative behaviors among all participants.

\section{Materials and Methods}

\subsection{Participants}

One-hundred 36 medical students (88 males and 48 females) at the University of Tsukuba, a national university in the Kanto region of Japan, participated in this program as part of a required course. The participants had little knowledge about suicide because they were second-year undergraduates of average age 19.96 years $(\mathrm{SD}=1.39$; range, 19-29 years).

\subsection{Contents of CAMPUS}

The main lecture is composed of three components, mental health literacy, antistigma, and crisis-management, each lasting about 20 minutes, created using a Power Point presentation (Microsoft Office PowerPoint 2016). A handout in "fill in the blanks" 
format is distributed to the students. In addition, CAMPUS includes two exercise sessions. In the first gatekeeper training session, students watch a 13-min gatekeeper training film showing student consultation scenes produced by the Ministry of Health, Labour and Welfare of Japan (2016). Next, they are divided into groups of three and perform a close listening exercise and discuss the importance of listening on outcome. This session requires about 30 minutes and is performed in combination with the second lecture on crisis management. In the second role-playing session, new groups of three perform two role-playing exercises in which they are assigned roles of a student with self-stigma (i.e., problems with mental illness, gender identity, and bullying) or suicide thoughts, a friend who listens to the student, and an observer. After the "student" reads a detailed scenario, he consults about his issues to the friend for a few minutes. Thereafter, the group discusses how the "student" can resolve these issues, how to proceed with consultation, and the mental issues faced by the "student." One role-playing session with discussion requires about 30 minutes. Facilitators trained in the use of CAMPUS monitor and facilitate these sessions. Students also complete various assessment scales before and after the program as detailed below.

\subsection{Procedures}

Prior to the intervention, we explained the importance of suicide prevention education for undergraduate medical students to the educational coordinator staff. We also obtained permission from the dean of the medical school to implement the suicide prevention education as a required class.

The CAMPUS was conducted using 3 class hours (one frame, 75 min) in July 2019, with mental health literacy and anti-stigma covered in the first hour, crisis-management and gatekeeper training in the second hour, and role-play sessions in the third hour. There was the lunch break between the first and second sessions. The main lecture was 
conducted by one author who is a psychiatrist at the university. The first author (a clinical psychologist) and three other psychiatrists acted as facilitators.

Self-reported questionnaires were administered before the program (pre-program), immediately after the program (post-program), and 6 months later (follow-up) in January 2020 to assess CAMPUS efficacy. The follow-up questionnaires were distributed after another class and collected during break time. The participants had no opportunity to participate in classes related to mental health and suicide for the intervening 6 months.

\subsection{Measures}

We used the following measures to evaluate the general efficacy of the CAMPUS.

\subsubsection{Suicide behaviors}

The Suicidal Behaviors Questionnaire-Revised (SBQ-R) is a 4-item self-report measure of suicide risk (Osman et al., 2001). The total score ranges from 3 to 18. The cut-off point for non-clinical samples is 7.

\subsubsection{Depression}

The Patient Health Questionnaire-9 (PHQ-9) is a 9-item self-report measure in which each item is scored from 0 (Not at all) to 3 (Nearly every day) as a measure of depression severity (Kroenke et al., 2001; Muramatsu and Ueshima, 2009). The total score ranges from 0 to 27 , and the cut-off point for non-clinical samples is 5 .

\subsubsection{Help-seeking intentions}

The General Help-Seeking Questionnaire (GHSQ) is a measure of help-seeking 
intentions for various resources when facing personal and emotional problems (Wilson et al., 2005). Each item is scored on a 7-point Likert scale ranging from 1 (Extremely unlikely) to 7 (Extremely likely). The help-seeking intentions for informal resources (intimate partner, friends, parents, and other relative/family members) and for formal resources (mental health experts, help lines, doctors, and ministers or religious leaders) were analyzed separately in the present research.

\subsubsection{Self-efficacy as a Gatekeeper}

The Gatekeeper Self-Efficacy Scale (GKSES) is a 9-item measure of confidence in gatekeeper skills (Takahashi et al., 2020). The students answered questions pertaining to gatekeeping skills including their knowledge of suicide prevention using a 7-point Likert scale from 1 (Not at all) to 7 (Extremely).

\subsubsection{Self-stigmatize attitude}

There is no single scale appropriate to measure self-stigma because the target differs markedly among individuals. Therefore, we used two psychological indices that are closely related to self-stigma. The first was a scale measuring self-acceptance of undesirable attributes by Tsukawaki, et al. (2009). The scale consists of eight items such as "I accept naturally my weakness." Each item is scored on a 5-point Likert scale from 1 (Agree) to 5 (Disagree). The second was the Japanese Self-concealment Scale (JSCS) (Larson and Chastain, 1990; Kawano, 2000), a 12-item scale assessing the tendency to actively conceal negative and distressing personal information from others. Each item is scored on a 5-poing Likert scale from 1 (Strong disagree) to 5 (Strong agree). Selfconcealment is related to suicidality (Hogge and Blankenship, 2020), suicidal behavior (Friedlander et al., 2012), and help-seeking attitudes for psychological professional services (Masuda et al., 2012). Positive changes on these two scales are considered 
indicative of self-stigma mitigation.

The SBQ-R and PHQ-9 were measured prior to intervention and at the 6-month follow-up, while the GKSES, GHSQ, self-acceptance, and JSCS self-concealment scales were measured at all three time points.

\subsection{Statistical Analyses}

We hypothesized that participants with strong suicidal ideation at baseline would demonstrate reduced ideation as measured by the SBQ-R if the program is effective, while no such change would be observed in those with low baseline ideation. Conversely, most subjects should demonstrate improved attitudes and competence related to suicide prevention. The SBQ-R has a minimum score of 3 and a cut-off of 7 , but 4 or more points indicates some ideation or a suicide-related episode compared to subjects who are not at any risk of suicide. Therefore, to test the above hypothesis, we divided participants into low- and higher-risk groups according to SBQ-R score (3 points vs. 4 or more).

The ratio of low-risk to higher-risk group participants was compared pre- and post-program using the McNemar test. Changes in other scale scores between low- and higher-risk groups were evaluated using repeated measures two-way analysis of variance (RT-ANOVA) with main factors time (pre-program, immediately postprogram, and 6-months post-program) and group (low-risk and higher-risk) with post hoc Bonferroni correction for multiple comparisons. The significance level of ANOVA was adjusted by Bonferroni's correction to $p<0.0083$ ( $p<0.05$ divided by six). When the main effect for time was significant, the Bonferroni test was used for post hoc analyses. When the time $\times$ group interaction was significant, a simple main effect test was performed. The effect size is expressed by partial $\eta^{2}\left(\eta_{\mathrm{p}}{ }^{2}\right)$. SPSS ver. 25.0 was used for all analyses. 


\subsection{Ethical considerations}

Written informed consent was obtained from all participants before the program. All students were informed of program aims and required attendance but that the questionnaires were optional. Follow-up e-mails were sent to students if the program SBQ-R or PHQ-9 score indicated cause for concern. The e-mails stated that they could always consult the University Health Center. The study was approved by the Medical Ethics Committee of the University of Tsukuba (No.1402-1).

\section{Results}

\subsection{Participant flow}

Among the 136 medical students attending the CAMPUS program as required, 121 (79 males and 42 females, 89.0\%) completed all questionnaires before and immediately after the program. The respondents were divided according to pre-program SBQ-R score $(M=3.87, S D=1.33)$ into a low-risk group of 66 students $(54.5 \%)$ scoring 3 points (minimum score) and a higher-risk group of 55 students $(45.5 \%)$ scoring 4 points or more (range, 4 to 9 points). In addition, 107 students (67 males and 40 females, $78.7 \%$ ) answered the follow-up questionnaires at 6 months post-program.

\subsection{Correlations between pre- and post-program psychometric scores}

The correlations between pre- and post-program test scores are shown in Table 1. Pre-program suicidal behavior as measured by the SBQ-R was positively but weakly correlated with depression severity as measured by the PHQ-9 $(r=0.25)$. Despite greater depression severity among participants at higher suicide risk (showing greater suicidal behavior), SBQ-R was negatively correlated with help-seeking intention for informal resources as assessed by the GHSQ $(r=-0.25)$. Also, SBQ-R was positively correlated 
with greater self-concealment score $(r=0.37)$. Poor self-acceptance was more strongly correlated with depression $(r=-0.34)$ than with suicidal behavior $(r=-0.19)$. Selfefficacy as a gatekeeper was correlated with help-seeking intentions for formal resources both pre-program $(r=0.33)$ and post-program $(r=0.39)$. Self-efficacy as a gatekeeper and self-acceptance were unrelated pre-program $(r=0.04)$, but significantly correlated post-program $(r=0.40)$.

\subsection{Changes in suicidal behavior}

The numbers of participants deemed low and higher risk for suicide according to SBQ-R scores pre- and post-program are shown in Table 2. Among 107 participants who completed all follow-up tests, 59 scored 3 on the SBQ-R pre-program (low-risk group) while 48 scored 4 or more (44.9\%, higher-risk group). At 6-months follow-up, a significantly greater number of participants scored $3(n=74)$ and significantly fewer scored 4 or more $(n=37,(34.6 \% ; p=.04$ by McNemar's test $)$. The highest score was 9 points pre-program, but it was 11 points after 6 months.

\subsection{Other longitudinal effects of the CAMPUS}

Average scores for all other pre-program, post-program, and follow-up tests as well as the results of two-way RT-ANOVA (main factors time and group) are shown in Table 3 . There were no significant time $\times$ group interactions.

Depression scores were below the clinical cut-off point for both the low-risk group and higher-risk group pre-program and at follow-up. The PHQ-9 depression severity scores were reduced in both groups at follow-up compared to pre-program baseline, but main effects of time and the interaction between time and group were not significant $\left(F(1,105)=0.26, p=.61, \eta_{\mathrm{p}}^{2}=.00 ; F(1,105)=0.01, p=.93, \eta_{\mathrm{p}}^{2}=.00\right)$.

There was a significant main effect of time on help-seeking intention for formal 
resources $(\mathrm{GHSQ}$ score $)\left(F(1.57,157.33)=14.86, p<.001, \eta_{\mathrm{p}}{ }^{2}=.13\right)$. Scores were higher post-program, but decreased significantly at follow-up. Help-seeking intentions for informal resources were greater at all time points in the higher-risk group, but the main effect of time was not significant $\left(F(1.68,163.08)=3.83, p=.03, \eta_{\mathrm{p}}^{2}=.04\right)$.

There was a significant main effect of time on gatekeeper self-efficacy $(F(2,208)=$ $142.45, p<.001, \eta_{\mathrm{p}}^{2}=.58$ ). Both groups demonstrated higher scores post-program, but scores decreased significantly from post-program to follow-up. Nonetheless, the score at follow-up was still higher than pre-program, indicating a long-term enhancement of gatekeeper self-efficacy.

There was also a main effect of time on self-acceptance score $(F(2,210)=7.53, p$ $<.001, \eta_{\mathrm{p}}^{2}=.07$ ), and both groups demonstrated significantly improved scores postprogram as well as at follow-up. Alternatively, self-concealment was not changed significantly $\left(F(1.74,182.80)=1.10, p=.33, \eta_{\mathrm{p}}^{2}=.01\right)$ although the low-risk group demonstrated higher scores post-program, whereas self-concealment declined postprogram in the higher-risk group.

\section{Discussion}

The purpose of this study was to examine the immediate and longer-term benefits of the CAMPUS for reducing suicidal behaviors and promoting preventative behaviors among university students. The proportion of participants deemed at higher risk for suicide according to SBQ-R score was significantly lower at 6 months post-program compared to baseline. Further, participants demonstrated improved self-acceptance and gatekeeper self-efficacy, two important suicide mitigation factors. Other suicide educational programs for university students have been shown to improve literacy and attitudes toward suicide, but have not been demonstrated to reduce suicidal ideation or behavior (Han et al., 2018; Katsumata et al., 2017; Indelicato et al., 2011; Kibler and 
Haberyan, 2008). In contrast, this relatively brief and easily delivered program tailored to the special needs of university students reduced suicidal behaviors (as revealed by the SBQ-R) at follow-up, strongly suggesting reduced suicide risk. Thus, CAMPUS can be effectively implemented on a smaller scale to reduce suicide risk, warranting studies on broader application throughout this and other institutions.

However, a few students had higher SBQ-R scores after 6 months, so CAMPUS as a primary suicide prevention program was not effective for all subjects, particularly those at highest risk at baseline in this study. Other programs may share this limitation. For example, students with severe suicidal ideation and suicide attempt were immediately taken to clinical assessment and referred to treatment, so they were excluded from an analysis of the YAM. (Wasserman et al., 2015). It is thus critical to underscore that the CAMPUS is a primary preventative measure and cannot replace targeted medical interventions. In our study, the number of high-risk students (SBQ-R $\geqq 7$ ) is small, so further research is needed on the effects of CAMPUS on high-risk students.

Educational contents and role-playing appeared to facilitate gatekeeper skills and self-acceptance regardless of suicide risk level, even after 6 months. Thus, the CAMPUS achieved one of its central aims, to enhance awareness of suicide risk in others, thereby increasing the likelihood that high-risk individuals are listened to and referred to mental health services.

Surprisingly, this reduction in suicidal behavior was not accompanied by marked changes in depression severity scores, possibly due to the relatively low baseline depression scores among the participants. Indeed, depression scores were significantly lower than in a previous study of medical students in Serbia (Miletic et al. 2015). However, the correlation between depression and suicide risk pre-program was weak. 
Mild depression may be relatively common among university students due to academic pressures and in some cases distance from family. These results suggest that the CAMPUS directly targets suicidal behaviors rather than reducing suicide risk by improving depression.

Importantly, CAMPUS also increased help-seeking intention for formal resources. Recognition of need and confidence in treatment benefits are important factors promoting help-seeking (Czyz et al., 2013; Downs and Eisenberg, 2012). Considering the positive correlation between help-seeking intention and gatekeeper self-efficacy, the students with high gatekeeper self-efficacy are more likely to consult formal resources for themselves and to refer others to these resources.

On the other hand, the CAMPUS did not improve self-concealment, implying that many participants were still reticent to discuss suicidal thoughts. Even though some participants role-played a suicidal person, the role-playing friends likely did not include anyone that the participant would actually consult. Those who can seek help from informal resources report greater well-being (Goodwin et al., 2016), so it is important to consult familiar people. Self-concealment may hinder help-seeking intentions for informal resources as low help-seeking intention for informal resources was associated with higher self-concealment in the present study. Surprisingly, self-concealment rating tended to decline in the higher-risk group but increased in the low-risk group immediately after the program. As the low-risk group had no suicidal ideation, they may have found that "it was surprisingly difficult or uncomfortable to tell people about suicidal ideation" during the role-playing session, resulting in greater self-concealment. In contrast, the higher-risk group with suicidal ideation may have found that "it was surprisingly safe to talk to people" by role-playing. However, the variance in selfconcealment scores was larger than other measures, indicating large inherent differences 
and responses to the role-playing exercise. Knowing a close friend or relation who has sought help can facilitate help-seeking behavior (Disabato et al., 2018), so help-seeking intention to informal resources may be improved by discussing experience of consulting close people in the CAMPUS exercises. Lindow et al. (2020) reported that teenage peers and school personnel did not promote help-seeking intention by YAM participants despite the program improving mental health knowledge and reducing the stigma surrounding mental illness; however, it did promote help-seeking behaviors. This suggests that students may consult informal resources even if they do not intend to seek help for themselves. For example, university students may seek or offer help for family members and friends as a result of the conversation. Therefore, it is necessary to measure help-seeking behaviors among CAMPUS participants in the future.

\section{Limitations}

This study has several limitations. First, although the questionnaire responses were voluntary, attending the CAMPUS was compulsory. Required participation may have raised gatekeeper self-efficacy and knowledge of suicide, but coercion in education can induce psychological reactance (Brehm, 1966). However, suicide prevention programs for elementary and junior high schools are similarly required, and required classes do not always have a negative impact on students. Nonetheless, it is necessary to consider how the form of education affects the program. In addition, academic skills could be an important factor influencing CAMPUS benefits, and participants in this study all demonstrated high academic capabilities. Therefore, program difficulty may need to be adjusted for other groups depending on the level of academic ability. We aim to distribute the CAMPUS program package to universities nationwide in the future, so additional studies are needed to implement a CAMPUS program applicable to 
university students of broad scholastic abilities.

\section{References}

Brehm, J.W., 1966. A Theory of Psychological Reactance. Academic Press, New York. Corrigan, P.W., Watson, A.C., 2002. The paradox of self-stigma and mental illness. Clin. Psychol. (New York). 9, 36-53.

Czyz, E.K., Horwitz, A.G., Eisenberg, D., Kramer, A., King, C.A., 2013. Self-reported barriers to professional help seeking among college students at elevated risk for suicide. J. Am. Coll. Health. 61, 398-406.

Disabato, D.J., Short, J.L., Lameira, D.M., Bagley, K.D., Wong, S.J., 2018. Predicting help-seeking behavior: the impact of knowing someone close who has sought help. J. Am. Coll. Health. 66, 731-738.

Downs, M.F., Eisenberg, D., 2012. Help seeking and treatment use among suicidal college students. J. Am. Coll. Health. 60, 104-114.

Friedlander, A., Nazem, S., Fiske, A., Nadorff, M.R., Smith, M.D., 2012. Selfconcealment and suicidal behaviors. Suicide Life-Threat. Behav. 42, 332-340. Goffman, E., 1990. Stigma: Notes on the Management of Spoiled Identity. Penguin, London [Ishigro, T., 2001. Tokyo: Serica syobo].

Goodwin, J., Behan, L., Kelly, P., McCarthy, K., Horgan, A., 2016. Help-seeking behaviors and mental well-being of first year undergraduate university students. Psychiatry Res. 246, 129-135.

Gorczynski, P., Sims-Schouten, W., Hill, D., Wilson, J.C., 2017. Examining mental health literacy, help seeking behaviours, and mental health outcomes in UK university students. J. Ment. Health Train. Educ. Pract. 12, 111-120.

Han, J., Batterham, P.J., Calear, A.L., Wu, Y., Xue, J., van Spijker, B.A.J., 2018.

Development and pilot evaluation of an online psychoeducational program for suicide prevention among university students: a randomised controlled trial. Internet Interv. 12, 111-120. doi: 10.1016/j.invent.2017.11.002

Harrod, C.S., Goss, C.W., Stallones, L., DiGuiseppi, C., 2014. Interventions for primary prevention of suicide in university and other post-secondary educational settings.

Cochrane Database System. Rev. 10, CD009439. doi:

10.1002/14651858.CD009439.pub2.

Hashimoto, N., Suzuki, Y., Kato, T.A., Fujisawa, D., Sato, R., Aoyama-Uehara, K.A., Fukasawa, M., Asakura, S., Kusumi, I., Otsuka, K., 2016. Effectiveness of suicide 
prevention gatekeeper-training for university administrative staff in Japan. Psychiatry Clin. Neurosci. 70, 62-70.

Hogge, I., Blankenship, P., 2020. Self-concealment and suicidality: mediating roles of unmet interpersonal needs and attitudes toward help-seeking. J. Clin. Psychol. doi: 10.1002/jclp. 22964

Indelicato N.A., Mirsu-Paun, A., Griffin, W.D., 2011. Outcomes of a suicide prevention gatekeeper training on a university campus. J. Coll. Stud. Dev. 52, 350-361.

Jain, A., Jain, R., Menezes, R.G., Subba, S.H., Kotian, M.S., Nagesh, K.R., 2012.

Suicide ideation among medical students: a cross sectional study from South India. Inj. Prev. doi: 10.1136/injuryprev-2012-040590n.5

Joffe, P., 2008. An empirically supported program to prevent suicide in a college student population. Suicide Life-Threat. Behav. 38, 87-103.

Jorm, A.F., Korten, A.E., Jacomb, P.A., Christensen, H., Rodgers, B., Pollitt, P., 1997. Mental health literacy: a survey of the public's ability to recognise mental disorders and their beliefs about the effectiveness of treatment. Med. J. Aust. 166, 182-186. doi: 10.5694/j.1326-5377.1997.tb140071.x

Katsumata, Y., Narita, T., Nakagawa, T., 2017. Development of a suicide prevention education program for university students: a single-arm pilot study. Asian J. Psychiatry. 30, 190-191.

Kawano, K., 2000. Correlational analysis among Japanese self-concealment scale, Kida's stimulus-seeking scale and self-reported physical symptoms. Jpn. J. Exp. Soc. Psychol. 40, 115-121.

Kibler, J., Haberyan, A., 2008. Suicide prevention training for resident assistants: Results of the Northwest training model. Based on a program presented at the ACA Annual Conference \& Exhibition, Honolulu, HI. http://counselingoutfitters.com/vistas/vistas08/Kibler.htm. (accessed 27 June 2020). Kroenke, K., Spitzer, R.L., Williams, J.B.W., 2001. The PHQ-9: Validity of a brief depression severity measure. J. Gen. Intern. Med. 16, 606-613.

Larson, D.G., Chastain, R.L., 1990. Self-concealment: conceptualization, measurement, and health implications. J Soc Clin Psychol. 9, 439-455.

Lindow, J.C., Hughes, J.L., South, C., Minhajuddin, A., Gutierrez, L., Bannister, E., Trivedi, M.H., \& Byerly, M.J., 2020. The Youth Aware of Mental Health Intervention: 
impact on help seeking, mental health knowledge, and stigma in U.S. adolescents. J. Adolesc. Health. 67, 101-107.

Manning, J., VanDeusen, K., 2011. Suicide prevention in the dot com era: technological aspects of a university suicide prevention program. J. Am. Coll. Health. 59, 431-433. Masuda, A., Anderson, P.L., Edmonds, J., 2012. Help-seeking attitudes, mental health stigma, and self-concealment among African American college students. J. Black Stud. $43,773-786$.

Miletic, V., Lukovic, J.A., Ratkovic, N., Aleksic, D., Grgurevic, A., 2015. Demographic risk factors for suicide and depression among Serbian medical school students. Soc. Psychiatry Psychiatr. Epidemiol. 50, 633-638.

Ministry of Health, Labour and Welfare of Japan, 2016. Kokoro no sign ni kiduitara: gakusei sodan hen [If you aware the mental sign: student consultation version]. https://www.youtube.com/watch?v=aOI62rKGxWQ\&list=PLMG33RKISnWjI0JFBIe6i 4eyYatP33rq0\&index $=24 \& \mathrm{t}=0$ s (accessed 4 August 2020).

Muramatsu, K., Ueshima K., 2009. Primary care practice and depression screening assessment tool: Patient Health Questionnaire-9 Japanese version," Mind and Body questionnaire.” Diagnosis and Treatment. 97, 1465-1473.

Osama, M., Islam, M.Y., Hussain, S.A., Masroor, S.M.Z., Burney, M.U., Masood, M.A., Menezes, R.G., Rehman, R., 2014. Suicidal ideation among medical students of Pakistan: a cross-sectional study. J. Forensic Leg. Med. 27, 65-68. doi: 10.1016/j.jflm.2014.08.006

Osman, A., Bagge, C.L., Gutierrez, P.M., Konick, L.C., Kopper, B.A., Barrios, F.X., 2001. The suicidal behaviors questionnaire-revised (SBQ-R): validation with clinical and nonclinical samples. Assessment. 8, 443-454.

Pattyn, E., Verhaeghe, M., Sercu, C., Bracke, P., 2014. Public stigma and self-stigma: differential association with attitudes toward formal and informal help seeking. Psychiatr. Serv. 65, 232-238.

Peltzer, K., Yi, S., Pengpid, S., 2017. Suicidal behaviors and associated factors among university students in six countries in the Association of Southeast Asian Nations (ASEAN). Asian J Psychiatry. 26, 32-38.

Rafal, G., Gatto, A., DeBate, R., 2018. Mental health literacy, stigma, and help-seeking behaviors among male college students. J. Am. Coll. Health. 66, 284-291. 
Sun, L., Zhou, C., Xu, L., Li, S., Kong, F., Chu, J., 2017. Suicidal ideation, plans and attempts among medical college students in china: the effect of their parental characteristics. Psychiatry Res. 247, 139-143. doi: 10.1016/j.psychres.2016.11.024 Takahashi, A., Tachikawa, H., Morita, N., Aiba, M., Shiratori, Y., Nemoto, K., Arai, T., 2020. Suicide prevention gatekeeper self-efficacy scale (GKSES). Crisis. 2020, 1-8. doi: $10.1027 / 0227-5910 / a 000698$

Tsukawaki, R., Higuchi, M., Fukada H., 2009. Relationships between humor expression and self-acceptance, aggression, and altruism. Jpn. J. Psychol. 80, 339-344.

Wasserman, D., Hoven, C.W., Wasserman, C., Wall, M., Eisenberg, R., Hadlaczky, G., Kelleher, I. Sarchiapone, M., Apter, A., Balazs, J., Bobes, J., Brunner, R., Corcoran, P., Cosman, D., Guillemin F., Haring, C., Iosue, M., Kaess, M., Kahn, J-P., Keeley, H., Musa, G.J., Nemes, B., Postuvan, V., Saiz, P., Reiter-Theil, S., Varnik, A., Varnik, P., Carli, V., 2015. School-based suicide prevention programmes: the SEYLE clusterrandomized controlled trial. Lancet. 385, 1536-1544.

Wilson, C.J., Deane, F.P., Ciarrochi, J., 2005. Can hopelessness and adolescents' beliefs and attitudes about seeking help account for help negation? J. Clin. Psychol. 61, 15251539.

Zheng, A., Wang, Z., 2014. Social and psychological factors of the suicidal tendencies of Chinese medical students. BioPsychoSocial Med. 8, 23. 
Table 1. Correlations among psychological scale scores for CAMPUS program participants $(N=121)$

\begin{tabular}{|c|c|c|c|c|c|c|c|}
\hline Scale & 1. SBQ-R & 2. $\mathrm{PHQ}-9$ & $\begin{array}{l}\text { 3. GHSQ } \\
\text { (formal) }\end{array}$ & $\begin{array}{l}\text { 4. GHSQ } \\
\text { (informal) }\end{array}$ & 5. GKSES & $\begin{array}{c}\text { 6. Self- } \\
\text { concealment }\end{array}$ & $\begin{array}{c}\text { 7. Self- } \\
\text { acceptance }\end{array}$ \\
\hline 2. & $.25^{* *}$ & - & & & & & \\
\hline 3. & $-.25^{* *}$ & $-.25^{* *}$ & - & $.43^{* * *}$ & $.24^{* *}$ & $-.21^{*}$ & .17 \\
\hline 4. & -.02 & -.03 & $.35^{* * *}$ & - & $.39^{* * *}$ & .10 & .04 \\
\hline 5. & .13 & -.05 & $.19^{*}$ & $.33^{* * *}$ & - & -.06 & $.40^{* * *}$ \\
\hline 6. & $.37^{* * *}$ & $.39^{* * *}$ & $-.24^{* *}$ & .08 & .11 & - & $-.28^{* *}$ \\
\hline 7. & $-.19^{*}$ & $-.34^{* * *}$ & .14 & .11 & .04 & $-.29^{* *}$ & - \\
\hline
\end{tabular}

The lower left grey zone shows the correlation coefficients pre-program, while the upper right shows the correlation coefficients post-program. ${ }^{*} p<.05,{ }^{* *} p<.01,{ }^{* * * *} p<.001$. 
Table 2. Distribution of SBQ-R scores $(N=107)$ before the CAMPUS program and at a 6-month follow-up

\begin{tabular}{|c|c|c|c|c|}
\hline \multirow[b]{2}{*}{ SBQ-R score } & \multicolumn{2}{|c|}{ Pre-program } & \multicolumn{2}{|c|}{ Follow-up } \\
\hline & $N$ & $\%$ & $N$ & $\%$ \\
\hline 3 & 59 & 55.1 & 70 & 57.9 \\
\hline 4 & 30 & 28.0 & 17 & 14.0 \\
\hline 5 & 8 & 7.5 & 11 & 9.1 \\
\hline 6 & 2 & 1.9 & 3 & 2.5 \\
\hline 7 & 4 & 3.7 & 2 & 1.7 \\
\hline 8 & 3 & 2.8 & 1 & 0.8 \\
\hline 9 & 1 & 0.9 & 2 & 1.7 \\
\hline 10 & 0 & 0 & 0 & 0 \\
\hline 11 & 0 & 0 & 1 & 0.8 \\
\hline$M(S D)$ & & 1.31) & & $.44)$ \\
\hline
\end{tabular}

SBQ-R: Suicidal Behaviors Questionnaire-Revised 
Table 3. Average psychological scale scores and ANOVA results for low suicide risk ("low risk") and higher suicide risk ("risk")

CAMPUS participants

\begin{tabular}{|c|c|c|c|c|c|c|c|c|c|c|c|c|}
\hline \multirow[b]{2}{*}{ Scale } & \multirow[b]{2}{*}{ Group } & \multirow[b]{2}{*}{$N$} & \multirow{2}{*}{$\begin{array}{c}\text { Pre } \\
M(S D)\end{array}$} & \multirow{2}{*}{$\begin{array}{l}\text { Post } \\
M(S D)\end{array}$} & \multirow{2}{*}{$\begin{array}{c}\text { Follow-up } \\
M(S D)\end{array}$} & \multicolumn{3}{|c|}{ Main effect of time } & \multicolumn{3}{|c|}{ Interaction } & \multirow[t]{2}{*}{ Differences } \\
\hline & & & & & & F-value & $p$ & $\eta_{p}^{2}$ & F-value & $p$ & $\eta_{p}^{2}$ & \\
\hline Depression & risk & 48 & $3.50(3.20)$ & - & $3.31(3.30)$ & & & & & & & \\
\hline $\begin{array}{l}\text { Help-seeking intention } \\
\text { for informal resources }\end{array}$ & low risk & 54 & $16.57(4.50)$ & $17.61(4.20)$ & $16.72(4.87)$ & 3.83 & .03 & .04 & 1.92 & .16 & .02 & \\
\hline \multirow{2}{*}{$\begin{array}{l}\text { Help-seeking intention } \\
\text { for formal resources }\end{array}$} & low risk & 55 & $10.49(4.75)$ & $13.00(5.06)$ & $11.44(5.11)$ & 14.86 & $<.001$ & .13 & 0.12 & .84 & .00 & pre $<$ post $^{* * *}$ \\
\hline & risk & 47 & $11.00(4.51)$ & $13.43(4.64)$ & $11.94(4.21)$ & & & & & & & post $<$ follow-up** \\
\hline \multirow{2}{*}{$\begin{array}{l}\text { Gatekeeper } \\
\text { self-efficacy }\end{array}$} & low risk & 58 & $25.91(10.11)$ & 43.41 (7.09) & $37.48(11.68)$ & 142.25 & $<.001$ & .58 & 0.16 & .85 & .00 & \\
\hline & risk & 48 & $25.52(11.29)$ & 44.15 (7.98) & $38.08(10.79)$ & & & & & & & pre $<$ follow-up ${ }^{* * *}$ \\
\hline Self-acceptance & risk & 48 & $24.75(5.83)$ & $26.67(5.19)$ & $25.94(4.81)$ & & & & & & & \\
\hline \multirow{2}{*}{ Self-concealment } & low risk & 59 & $35.97(13.57)$ & 40.14 (12.72) & $36.39(14.25)$ & 1.10 & .33 & .01 & 4.93 & .01 & .04 & \\
\hline & risk & 48 & $44.15(15.66)$ & $40.75(15.14)$ & $41.08(15.84)$ & & & & & & & \\
\hline
\end{tabular}

Note. Significance level of main effects and interaction $=p<.0083$. Pre $=$ pre-program, Post $=$ post-program, Follow-up $=6$ months after the program. ${ }^{* *} p<.01,{ }^{* * *} p<.001$ 